\title{
Open Software and Art: A Tutorial
}

\author{
Letizia Jaccheri \\ Department of Computer and Information Science, NTNU, Trondheim 7491, Norway \\ letizia@idi.ntnu.no \\ http://letiziajaccheri.com
}

\begin{abstract}
The tutorial is devoted mainly to $\mathrm{PhD}$ students, researchers, educators, and artists who are interested to learn, practice, and reflect about software tools for creativity and art. In this work, we chose to focus on open source software and its intersection with art. This choice is motivated by significant related work in open source software and art and available software for art like Processing, Arduino, and Scratch. Four research questions will shape the discussion: 1) Development or use of software? 2) Who are the stakeholders? 3) How to evaluate art and technology works? 4) Are there feelings beyond creativity and amusement one should aim at when designing art and technology expressions?
\end{abstract}

Keywords: Art and Technology, Open Source Software, Creativity, Arduino, Processing, Scratch.

\section{Introduction}

The general research question that shapes our tutorial is: How can we increase knowledge about the intersection between software and art? This question has guided the work of researchers, artists, and technologist in the last 40 years [3], [8], [4]. Given that software is getting increased power on the way people experience art and culture, increased knowledge about the intersection of software and art benefits cultural industries, which produce media for publishing, museums, and entertainment. More specifically, increased knowledge about software and art provides both better technology platforms for cultural industries and better conceptual models for decision makers. Knowledge about software tools for creativity and art is important for artists, engineers, students, educators, and researchers in art and technology [11] [13] [16]. In the proposed tutorial, we chose to focus on open source software and its intersection with art and available software for art like Processing, Arduino, and Scratch. Moreover, we will describe examples of artistic expressions based on these technologies.

This proposal has the following structure. Section 2 provides information about the tutorial implementation and the teacher. Section 3 provides the background literature which addresses issues related to software and art. Section 4 presents four main research questions that will shape the tutorial. Section 5 gives a conclusive summary. 


\section{Information about the Tutorial and the Teacher}

The tutorial is devoted mainly to $\mathrm{PhD}$ students and researchers in art and technology. Educators and artists who are interested to learn, practice, and reflect about software tools for creativity and art are also invited. We aim at twelve to twenty participants.

The tutorial will last one full day and it will be organized as a combination of a learning session and hands-on applied sessions. The learning session consists of lectures by the teacher on the theme presented in this proposal. In addition, there will be three small hands-on applied creative sessions in which the participants will be able to experiment with Scratch [10], Arduino [5], and Processing [6].

Participants will have to bring their laptop (Windows, Linux, or Mac). It is an advantage if the participants have already installed the three tools, but this is not mandatory. No practical programming knowledge is required. Participants will be able to work in small groups and to improvise presentations. Pictures will be used as a mean to record and reflect about the creative activities. The ArTe blog [14] links to a set of reusable resources, among which three master level and a $\mathrm{PhD}$ course in the field of software and art. The blog also documents a set of creative workshops that have been offered by the same teacher and her group during the last years. This documentation includes pictures, videos, and code fragments to be reused.

In this tutorial, we chose to focus on open source software (OSS) and its intersection with art. This choice is motivated by significant related work in OSS and art and available OSS for art like Processing [11], [6], Arduino [5], and Scratch [10].

Letizia Jaccheri is a professor at NTNU, Norway. She has twenty years' experience of teaching and researching and she has been involved in the supervision of more than $12 \mathrm{PhD}$ students. Concerning the theme of software and art, she teaches three master level (one theory based and two practice based [9] and a PhD level course. She has published book chapters and papers and has given presentations at several universities in Europe. Her motivation for developing this tutorial is to test the bulk of knowledge and practical learning methods with an International audience. Lastly the aim is to connect to the ICEC community.

\section{Background}

Artists need software technology for creating and evolving their artwork [16]. Theoreticians aim at understanding the consequences of software to art practices [7]. Technologists see the contact with artists as a source for innovation [8]. Computer science researchers recognize the importance of new media art as a legitimate research field [12]. Tools for creativity are a subject of study within computer science research. 'New media artists realize their desire for personal expression with powerful development environments that support animation, music, or video editing tools' [13].

The inner joy of creation has often been identified as an attribute of the open source software developer culture, bringing it, according to Castells [2] close to the world of art. Castells anticipates art as a growing area of the Internet, stating that 'open source art is the new frontier of artistic creation'. For Castells, the Internet not only serves as a means for distribution of artifacts, but also serves as a platform for a process that aims to create new artistic artifacts. 
A software system is open source if its code is available to everybody for inspection, use, and modification. Users of OSS are not paying customers but software co-developers. Use and further release of modified version of an OSS system are regulated by a license. OSS is much more than the possibility to change the code. OSS projects are associated with communities of users and developers. The degree of activeness of the community is crucial for an OSS project. Each user of an active community is not isolated but part of a community. Members of each community are connected and help each other via mailing lists, forum and IRC channels.

\section{Research Questions}

There are at least four questions to stimulate reflection on the relationship between computing and art.

RQ1: Development or use? In the computing world, the relation with art is mostly visible in the subfield of Human Computer Interaction. The novelty of our work is that we look at the intersection of software engineering and art [16]. While HCI is concerned with the use of computer systems, software engineering is concerned with the development and evolution of software. We will point to events in which the users have been empowered to be the producers of fragments of OSS code while developers, artists, and researchers act as enablers and observers during the events. Developers and artists are themselves actors of the creative process that has the purpose of designing the events and producing the artifact that serve as components to the event.

RQ2: Who are the stakeholders? In previous work [15], [16], we have identified the importance of a stakeholder model. The work reported in this tutorial goes a step further by giving concrete examples of art and software projects and the involvement of the different stakeholders. In the tutorial we will discuss how the roles of artist, researcher, developer, audience are important to be able to plan and reflect about art and software projects. At the same time, these roles are not fixed and each person can play in the context of the same project or of different projects different roles.

RQ3: How to evaluate works in this intersection? Evaluation methods of user experience require a search for a balance between the research goal and the artistic goal. The criteria for successful art events can be different from the criteria for successful research. While artists may see evaluation activities as disturbing, evaluation activities have to be integrated in a way they become acceptable from an aesthetics point of view.

RQ4: Are there feelings beyond creativity and amusement we should look at? An important dimension is the role of creativity in the intersection of software and art. A superficial view of the intersection may lead to a model that categories artists as creative and engineers and researchers as analytical. 'Creativity is often thought of as a trait exclusive to artists and primarily an individual activity' [1]. Another interesting point of our work is that we dare to give words to other emotional states rather than playfulness and creativity when describing the relations between users and technology. In our cases we will describe romantic, contemplative, and boring situations. 


\section{Conclusions}

A full day tutorial will be organized as a combination of a learning session and handson applied sessions with OSS tools for creativity. The learning sessions will be grounded in both related literature and the research experience of the teacher. The hands-on sessions will have the goal to let the participants experiment with software tools for creativity and to work in small groups of participants. This will enable the participants to get to know each other in a creative and motivating atmosphere. Our experience with similar creative sessions, especially with the Scratch tool, tells that participants will be able to create and share their contributions.

\section{References}

1. Aragon, C.R., et al.: A tale of two online communities: fostering collaboration and creativity in scientists and children. In: Proceeding of the Seventh ACM Conference on Creativity and Cognition

2. Castells, M.: Rise of The Network Society (Castells, M:. Information Age 1), vol. 1. Wiley, Chichester (1996)

3. Edmonds, E.A., et al.: The studio as laboratory: Combining creative practice and digital technology research. Intl Journal of Human Computer Studies 63, 4-5 (2005)

4. Fishwick, P.: Aesthetic Computing. MIT Press, Cambridge (2006)

5. Arduino Group: Arduino web site (March 2011), http: / / arduino. CC

6. Processing Group: Processing web site (March 2011), http: / / processing . org

7. Halonen, K.: Open source and new media artists. Human Technology - An Interdisciplinary Journal Humans in ICT Environments 3 (2007)

8. Harris, C. (ed.): Art and innovation: the Xerox PARC Artist-in-Residence program. MIT Press, Cambridge (1999)

9. Jaccheri, L., Sindre, G.: Software engineering students meet interdisciplinary project work and art. In: IV, pp. 925-934. IEEE Computer Society, Los Alamitos (2007)

10. Scratch Group: Scratch web site (March 2011), http: / / scratch . mit. edu

11. Noble, J.: Programming Interactivity: A Designer's Guide to Processing, Arduino, and Openframeworks, 1st edn. O’Reilly Media, Sebastopol (2009)

12. Oates, B.J.: New Frontiers for Information Systems Research: Computer Art as an Information System. European Journal of Information Systems 15 (2006)

13. Shneiderman, B.: Creativity support tools accelerating discovery and innovation. Communication of the ACM 50(12), 20-32 (2007)

14. ArTe Team: Arte's blog and content management system (March 2011), http: / / www . artentnu. com

15. Trifonova, A., Ahmed, S.U., Jaccheri, L.: SArt: Towards innovation at the intersection of software engineering and art. In: Proceedings of the 16th International Conference on Information Systems Development, pp. 29-31. Springer, Heidelberg (2007)

16. Trifonova, A., Jaccheri, L., Bergaust, K.: Software engineering issues in interactive installation art. International Journal on Arts and Technology (IJART) 1(1), 43-65 (2008) 